implicitly relying on enough other children being vaccinated to maintain herd immunity'. This has the strategic structure of a social dilemma, because if all parents followed this reasoning, everyone could end up worse off than if they all behaved cooperatively.

A similar social dilemma is devastating UK fish stocks: overfishing destroyed British herring fisheries long ago and is now causing terminal decline in other fish stocks in the English Channel, the North Sea and the Baltic. Anyone who makes a living by fishing is motivated to catch as many fish as possible, because restraint is pointless if enough others are exercising restraint, and is futile if they are not. But then fish are driven to extinction and everyone is worse off than if they had all restrained themselves cooperatively.

Bill Hamilton's theory of 'inclusive fitness', or kin selection, explains the evolution of cooperation among genetically related individuals. It can explain the extreme self-sacrificing cooperation of female social Hymenoptera, who have $75 \%$ of their genes in common, but cannot explain cooperation among non-relatives. Robert Trivers's theory of reciprocal altruism shows how cooperation between non-relatives can evolve if its cost is small and is outweighed by favours returned in the future. These two theories go some way towards explaining how cooperation evolved, but neither can explain human cooperation in unrepeated interactions between strangers.

To fill this gap, Ernst Fehr and Simon Gächter introduced in 2000 a version of the theory of strong reciprocity incorporating the 'altruistic punishment' of non-cooperators. Moral Sentiments and Material Interests is devoted to their theory's biological, anthropological, economic and social ramifications and related ideas. An introductory chapter is followed by three on the behavioural ecology of cooperation, four on modelling and testing strong reciprocity, and five on reciprocity and social policy, all by researchers in the vanguards of their fields.

According to the theory, cooperation is necessary for the provision of public goods, and the punishment of non-cooperators, or freeriders, is itself a public good - a service provided for the benefit of the whole community. Such punishment is altruistic because it is costly to those who administer it, as it takes time and energy and invites retaliation. Fehr and Gächter have provided persuasive experimental evidence, reviewed in this book, that cooperation flourishes when punishment is possible and breaks down when it is not.

A problem not addressed in the book is that, because altruistic punishment is costly, natural selection should tend to eliminate it. Failure to punish defectors must presumably be treated as second-order defection, itself subject to sanctions from other group members. But what about sanctions against third-order defectors who neglect to punish second-order defectors, and so on? This is an infinite regress

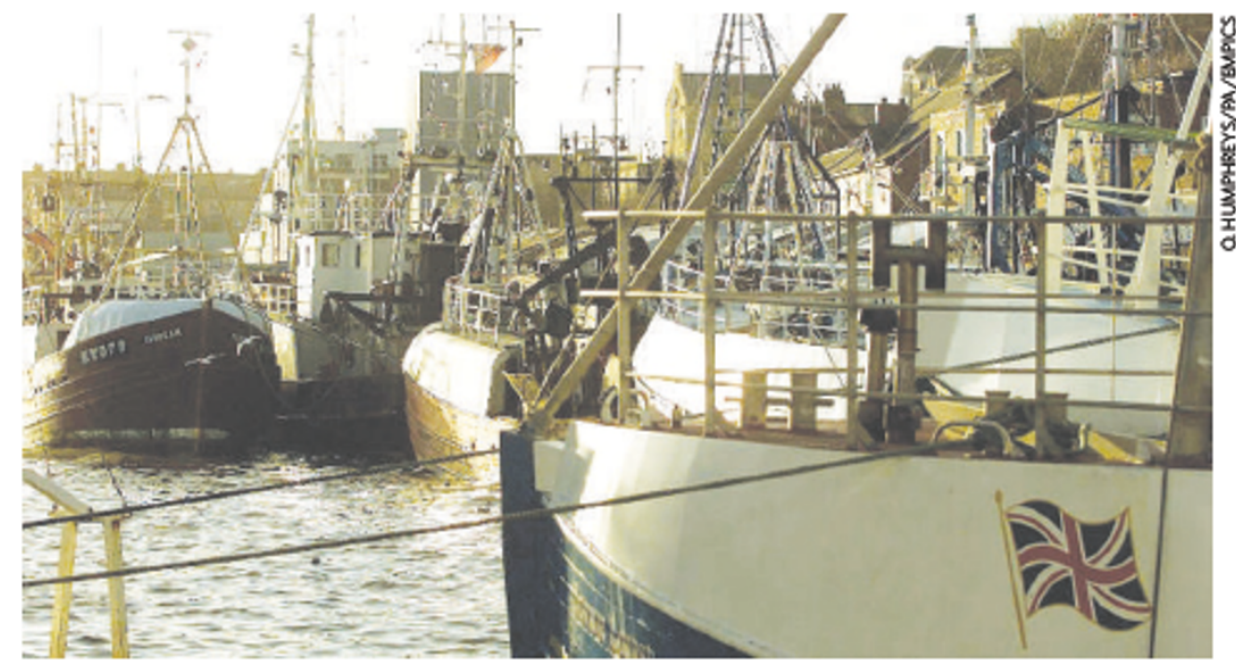

Tragic commons: North Sea fishing boats sitidle, thanks to rational economic decision-making.

that becomes less credible with the addition of each successive layer. Strong reciprocity is an important and illuminating discovery, but we seem to have replaced the problem of explaining cooperation with that of explaining altruistic punishment.

The book provides a superb interdisciplinary synthesis of cooperation as explained by strong reciprocity and associated phenomena. Other explanations of cooperation in unrepeated interactions between strangers hardly get a look in, however. The most important is Richard Alexander's theory of 'indirect reciprocity', according to which people use observations of direct reciprocity between others when deciding how to act towards them in the future. People benefit by cooperating, even in one-off encounters with strangers, because cooperation enhances one's reputation for cooperativeness and elicits reciprocal cooperation from others. This is a powerful theory, supported by evidence from computational and experimental studies, but Moral Sentiments and Material Interests mentions it only in passing. Andrew M. Colman is in the School of Psychology, University of Leicester, Leicester LE1 7RH, UK.

\title{
Back to the drawing board?
}

Knowing: The Nature of Physical Law
by Michael Munowitz
Oxford University Press: 2006. 432 pp

Ł19.99, \$35

\section{David Lindley}

Among the many services that Michael Faraday rendered to science was his innovation of illustrating theoretical ideas by means of pictures and diagrams. For Faraday, who knew next to no mathematics but had a powerful visual imagination, the image was the concept. But as his successors dressed his ideas in the finery of nineteenth-century mathematics, we have come to think of mathematics as the essence of theory, with pictures being merely a helpful adjunct. Attempting to push the pendulum back a little, Michael Munowitz has written an account of modern physics that Faraday might appreciate, using no mathematics (well, all right, there is some arithmetic) but plenty of illustrations. His mixed success shows, rather sadly perhaps, how far theory has moved beyond our ability to capture it in an easily grasped visual form.

Munowitz plunges in with an opening chapter devoted to the four elementary forces of nature. Gravity holds the Solar System together, and electromagnetic forces do much the same thing for atoms. Going to a still smaller scale, we see how there must be a strong nuclear force to prevent positively charged protons from bursting out of the nucleus. This is nicely done. In each case, we perceive the force through its ability to control a physical system.

But then comes the pesky weak nuclear force, or rather interaction. The change of word betrays the problem. If there is some visualizable structure that the weak interaction holds together, I can't think of it, and neither can Munowitz. Instead, he talks about radioactive decays that the weak interaction engenders and the connection to something that might be called a force is quietly dropped. I don't mean this as a criticism so much as a recognition of how difficult a task Munowitz has set himself.

Where Munowitz succeeds admirably, however, is in his recurrent emphasis on the way that principles of invariance and symmetry dictate the shape of natural law. Insistence that physics cannot depend on absolute velocity - there is no such thing - leads to the conservation law for momentum. Likewise, the impossibility of absolute time takes us (not so obviously) to the conservation of energy. In neither case can Munowitz make 
the connection precise, but he manages to make the reader feel that the conservation laws are not ex cathedra pronouncements but derive from eminently sensible assertions about the way the Universe is constructed.

A later chapter builds on these arguments to show how local gauge invariance of the electromagnetic field, for example, demands a force to make sure that particles behave as they must towards each other. And the tautological principle that an observer cannot tell the difference between identical quantum objects leads into a nice account of Pauli's principle and exchange forces. In these sections Munowitz splendidly demonstrates how a handful of bedrock principles underlie many apparently different areas of physics. The avoidance of mathematics is a real advantage here, forcing attention on to broad concepts rather than specific rules.

From time to time I had the nagging feeling that this book may be illuminating in the way that Richard Feynman's celebrated lectures are - by striking flashes of intellectual enlightenment in readers who have already learned physics the old-fashioned way. A young Fara- day of our times, looking to this book for an education, would in the end be obliged to take many of the conclusions on trust. And in a few chapters, Munowitz fumbles the conceptual continuity, delivering catalogues of things that the dutiful reader ought to know. But for any high-school student or undergraduate who is losing sight of the forest on account of all the trees, Knowing at times offers a persuasively harmonious view of the physicist's way of looking at the world.

David Lindley is a freelance writer based in

Alexandria, Virginia, USA.

\section{High impact}

\section{From protons to galaxies, Cosmic Collisions shows us what happens when things go bump.}

\section{Michael Hopkin}

Our galaxy is big. But it's due to get a lot bigger: in a few billion years, the Milky Way will slam into its nearest neighbour, the Andromeda Spiral, eventually creating a super-sized stellar clump. And visitors to the Hayden Planetarium at New York's

American Museum of Natural History can now see a preview of the monumental event as it is likely to unfold.

The scene is the denouement of Cosmic Collisions, a \$3-million film that reveals the power of impacts great and small to shape

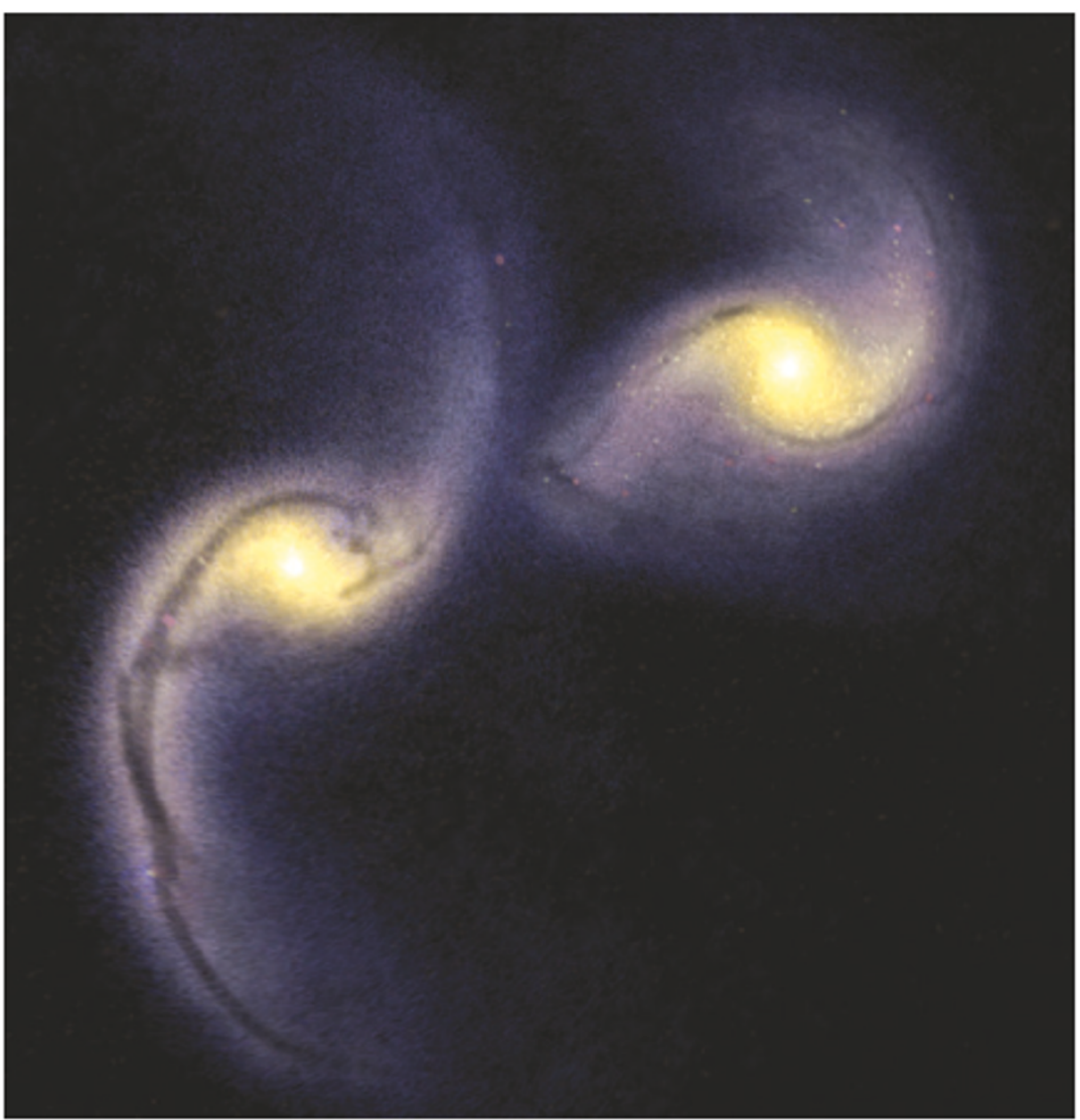

Forthcoming attraction: a simulation of the Milky Way's merger with Andromeda (left).

our world and the Universe. The projectalso showcases the prodigious supercomputing power now available to astrophysicists the largely computer-generated movie visualizes some of theorists' most advanced simulations of cosmic processes.

The film focuses on impacts across a mind-boggling range of scales, from the proton collisions that power the Sun, to the asteroid smash that may have done for the dinosaurs, to the pas de deux of galaxies played out over millions of years.

The team behind the film, including the

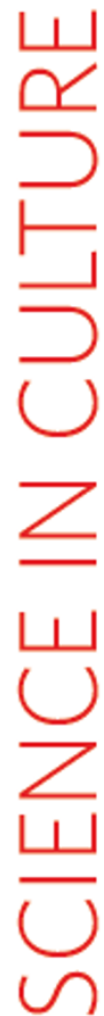

museum's astrophysics department, NASA and more than 25 researchers from around the world, based their pictures on supercomputer simulations and satellite images.

The starry sky, created using a virtual map called the Digital Universe, forms a backdrop to the film's opening, over which the gravelly tones of Robert Redford informus that, although space might look tranquil, its myriad collisions make it a violent place.

This becomes clear during a scene in which the serenly drifting Earth is suddenly slammed by a planet-sized body that seems to appearfrom nowhere. This event really happened, 4.5 billion years ago, and in the space of a month it gave us our Moon. The film draws on simulations of lumps of debris being drawn together by gravity to show us how this remarkably rapid process occurred.

The film culminates with the climactic sequence in which two galaxies, each containing hundreds of billions of stars, meet. The sequence, which lasts less than a minute but in which every second represents 40 million years, is the product of more than 40,000 hours of computing time.

The galactic clash is based on simulating the behaviour of individualstars within each cluster, and comparing the results with data on real galactic collisions. Transforming that information into a visual sequence was the work of yetmore supercomputers.

As for the event itself, the collision won't cause much disruption to any civilizations still around. As Redford reassures us, "Stars and planets in these galaxies won't actually collide. They're much too far apart.

Scientists think they'll simply slide past one another." Nevertheless, you'll have to go a lot further than New York to get such a grandstand view of the real thing.

Cosmic Collisions is showing at the Hayden Planetarium, American Museum of Natural History, New York.

Michael Hopkin writes for news@nature.com. 